\title{
Vasorelaxant activity of indole alkaloids from Tabernaemontana dichotoma.
}

\begin{abstract}
The aim of this study was to search for bioactive natural products from medicinal plants targeting vasorelaxant activity and we found the methanol extract from bark of Tabernaemontana dichotoma showed vasorelaxant activity on rat aorta. We isolated eight indole alkaloids including 10-methoxyalstonerine (1), a new macroline type indole alkaloid, from bark of T. dichotoma. These were respectively identified as 10-methoxyaffinisine (2), lochnerine (3), cathafoline (4), (-)-alstonerine (5), 19,20-dehydro-10-methoxytalcarpine (6), alstonisine (7), and alstonal (8) based on spectroscopic analysis. Among them, sarpagine type (2 and 3), akuammiline type (4), and macroline oxindole type (7 and 8) showed potent vasorelaxant activity. Mechanism of action on vasorelaxant activity of 10-methoxyaffinisine (2), cathafoline (4), and alstonisine (7) was clarified. Effects of 10-methoxyaffinisine (2), cathafoline (4), and alstonisine (7) were partially mediated the NO release from endothelial cells. Furthermore, 10-methoxyaffinisine (2) and alstonisine (7) attribute to the inhibitory effect of VDC and ROC, and cathafoline (4) have inhibitory effect on Ca2+ influx via ROC. In addition, 10-methoxyaffinisine (2) as a major compound from bark of $\mathrm{T}$. dichotoma showed hypotensive effect on normotensive rats in vivo.
\end{abstract}

Keyword: 10-methoxyaffinisine; 10-methoxyalstonerine; Apocynaceae; Hypotensive effect; Rat aortic artery; Tabernaemontana dichotoma; Vasorelaxant effect. 